\title{
Malignant Melanoma Arising in Association with Sinonasal Mela- nosis: A Case Report and Review of the Literature
}

\author{
Eric Ewing ${ }^{1}$, Jeffrey B Jorgensen ${ }^{2}$, Benjamin F Erhardt ${ }^{2}$ and Lester J Layfield ${ }^{1 *}$ \\ ${ }^{1}$ Department of Pathology and Anatomical Sciences, University of Missouri, USA \\ ${ }^{2}$ Department of Otolaryngology - Head and Neck Surgery, University of Missouri, USA
}

*Corresponding author: Lester J Layfield, MD, Professor and Chair, Department of Pathology and Anatomical Sciences, University of Missouri, One Hospital Drive, M263 Medical Sciences Building, Columbia, MO 65212, USA, Tel: (573)-8821201, Fax: (573)-884-4612, E-mail: layfieldl@health.missouri.edu

\begin{abstract}
Background: Malignant melanomas of the nasal mucosa represent approximately $1 \%$ of all melanomas and reported examples often arise in areas of pigmentation of the nasal mucosa. Melanin pigmentation has been reported to occur in the nasal glands and dendritic cells but melanomas arising from areas of pigmented (melanocyte containing) nasal glands have not been reported to our knowledge.

Case presentation: A primary sinonasal malignant melanoma arising in association with melanosis of the nasal mucosa and seromucinous glands is reported in a seventy-six-year-old Caucasian male. Focal pigmentation of the seromucinous glands and surface epithelium of the nasal septum was present and associated with immunoreactivity for HMB-45 and melan-A and a positive Fontana-Masson reaction. A malignant melanoma was present, arising within the area of the melanosis.

Conclusions: The present case demonstrates that nasal melanomas may arise from areas of nasal seromucinous glands containing melanocytes and melan pigment.
\end{abstract}

\section{Keywords}

Nose, Nasal glands, Melanoma, Pigment, Melanin

\section{Background}

Pigmentary disorders of the nasal mucosa and submucosal glands are rare and include melanocytic oncocytic metaplasia [1,2], melanocytic hyperplasia of the nasal respiratory epithelium, and primary malignant melanoma [3]. Intranasal malignant melanoma represents approximately $1 \%$ of total malignant melanomas arising at all body sites [4]. Dendritic melanocytes have been identified within the intranasal respiratory epithelium and may represent the site of origin for many if not most nasal malignant melanomas [4-6].
Melanotic oncocytic metaplasia shows coexistence of oncocytic metaplasia and melanin pigmentation within the same gland $[1,7,8]$. The precise origin of the melanin pigment within the oncocytic epithelium is unclear. Melanocytes are known to exist within the stroma and epithelium of the nasal cavity and paranasal sinuses $[5,6,9]$ and these cells may be the source of the melanin pigment within the oncocytic epithelium [7]. Cases of melanotic oncocytic metaplasia demonstrate dendritic melanocytes which are S-100 positive but HMB-45 negative. To date melanotic oncocytic metaplasia has not been associated with the development of malignant melanoma $[1,2,10,11]$. In this review, we report a case of melanosis of nasal seromucinous glands arising within a Caucasian male and associated with a malignant melanoma primary within the nasal cavity. In our case, the seromucinous glands did not show oncocytic metaplasia, but melanin was present in a pattern similar to that reported in melanotic oncocytic metaplasia.

\section{Case Presentation}

This report was approved by the Veteran's Administration Institutional Review Board.

A 75-year-old man presented with a one month history of left ear pain, drainage and decreased hearing in the left ear. In addition, he reported chronic left nasal congestion with a constant inability to breathe through his left nostril. He was recently treated for acute sinusitis at an outside hospital which resolved after antibiotic therapy, however, his ear symptoms and nasal conges-

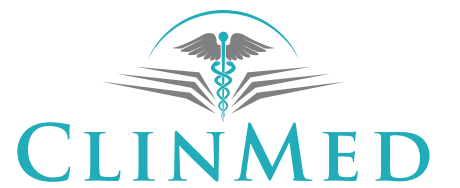

INTERNATIONAL LIBRARY
Citation: Ewing E, Jorgensen JB, Erhardt BF, Layfield LJ (2017) Malignant Melanoma Arising in Association with Sinonasal Melanosis: A Case Report and Review of the Literature. Int J Pathol Clin Res 3:058. doi.org/10.23937/2469-5807/1510058

Received: May 06, 2016: Accepted: August 31, 2017: Published: September 02, 2017

Copyright: (c) 2017 Ewing E, et al. This is an open-access article distributed under the terms of the Creative Commons Attribution License, which permits unrestricted use, distribution, and reproduction in any medium, provided the original author and source are credited. 
tion persisted. The patient denied epistaxis, but did report small amounts of hemoptysis daily.

A flexible nasolaryngoscopy was performed which demonstrated a small mass extending off the posterior edge of the left septum with associated dark mucosa (Figure 1) extending beyond the mass up to the level of the superior turbinate to the left nasal floor and on the right around the choana to the right nasal floor. In addition, there was significant left nasal septal deviation. A biopsy of the nasal mass demonstrated a spindle cell melanoma. A posterior septectomy was performed including bilateral

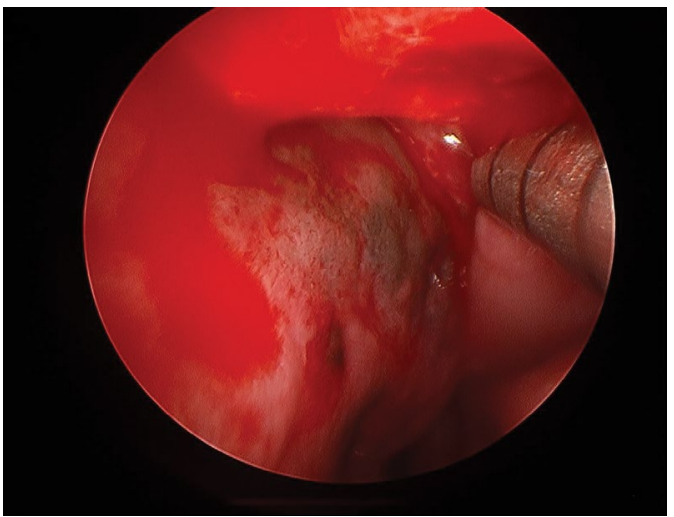

Figure 1: Flexible nasolaryngoscope image of pigmented area and mass at posterior edge of nasal septum.

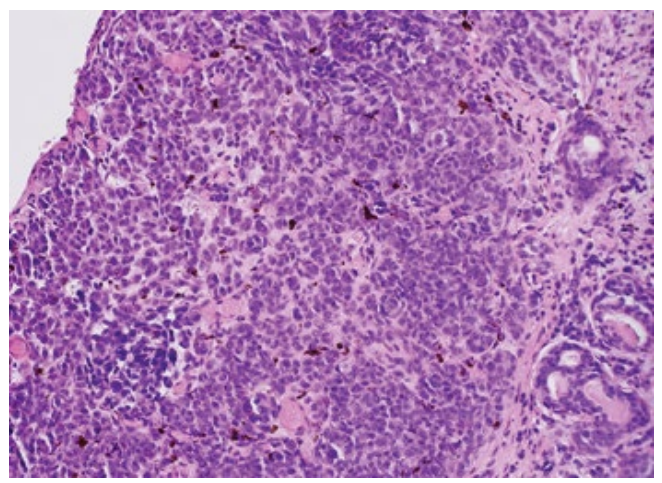

A superior and right middle turbinectomy in addition to excision of the sphenoid face and bilateral nasal floor mucosa.

\section{Pathologic Findings}

The resection specimen was composed of a $3.7 \times$ $3.5 \times 0.5 \mathrm{~cm}$ aggregate of soft tissue, bone and cartilage containing areas of blue-brown discoloration of the mucosal surface.

Histologic evaluation disclosed a pigmented focally ulcerated mass surrounded by a zone of pigmentation within both the mucosa and some of the seromucinous glands. The mass was composed of atypical oval to polygonal cells with scant to modest amounts of cytoplasm containing scattered brown pigment. Morphologically, the neoplasm was consistent with malignant melanoma (Figure 2A and Figure 2B). No in situ component of the melanoma was seen. The mucosa over the mass was ulcerated and the neoplastic cells invaded the underlying soft tissues and surrounded and destroyed the entrapped seromucinous glands. At the periphery of the mass, the seromucinous glands contained pigment morphologically identical to that seen in the melanoma (Figure $3 \mathrm{~A}$ ). The pigment lay in the cuboidal to low column cells lining the lumen of the glands (Figure $3 B$ ). Immunohistochemical staining for $\mathrm{S}-100$ protein, HMB-45 and melan A showed the glandular cells to be immuno-

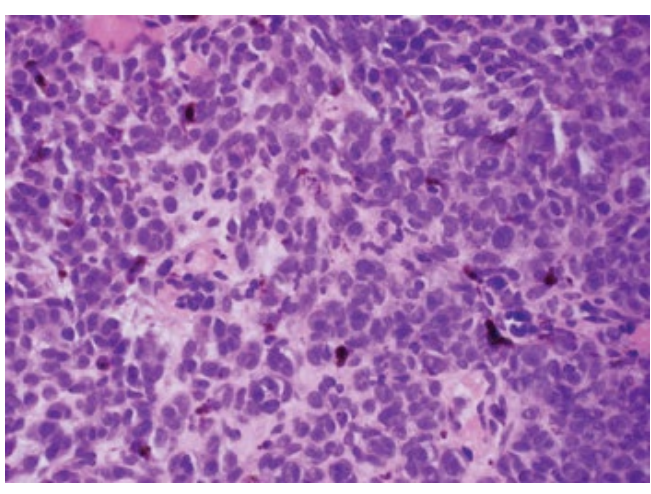

B

Figure 2: A) Photomicrograph of mass lesion composed of atypical oral and polygonal cells representing malignant melanoma ( $H+E$, $\mathrm{X} 200)$; B) The oval and polygonal cells demonstrate nuclear hyperchromasia and membrane irregularities $(\mathrm{H}+\mathrm{E}, \mathrm{X} 400)$.

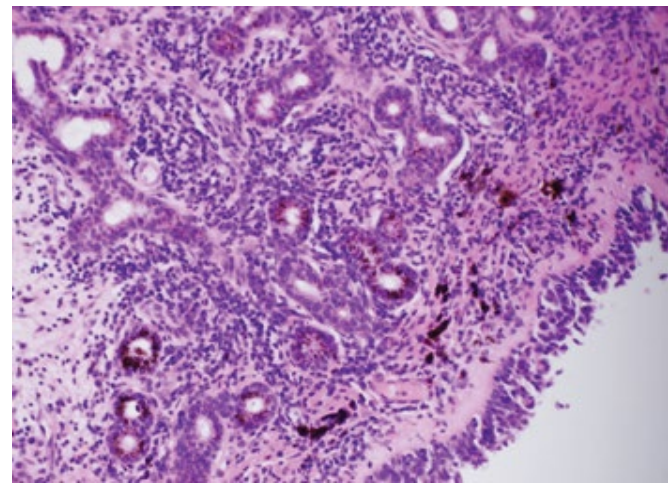

A

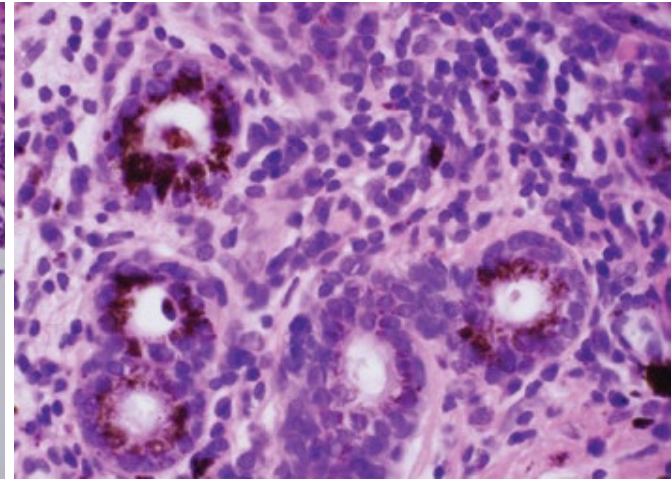

B

Figure 3: A) Seromucinous glands of nasal mucosa containing melanin pigment (H+E, X200); B) Prominent melanosis of epithelium of glands $(\mathrm{H}+\mathrm{E}, \mathrm{X} 600)$. 


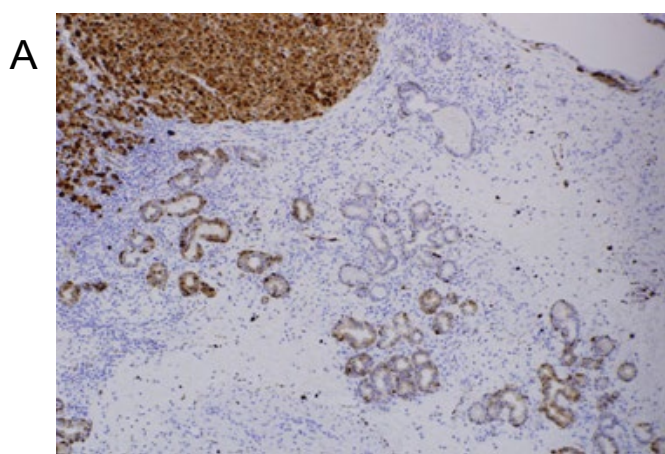

$\mathrm{B}$

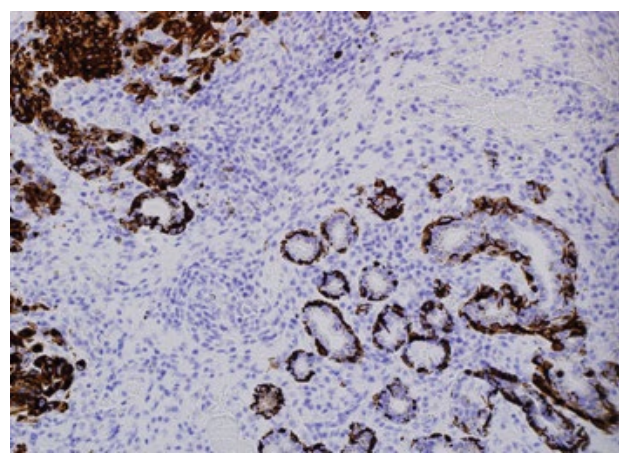

C

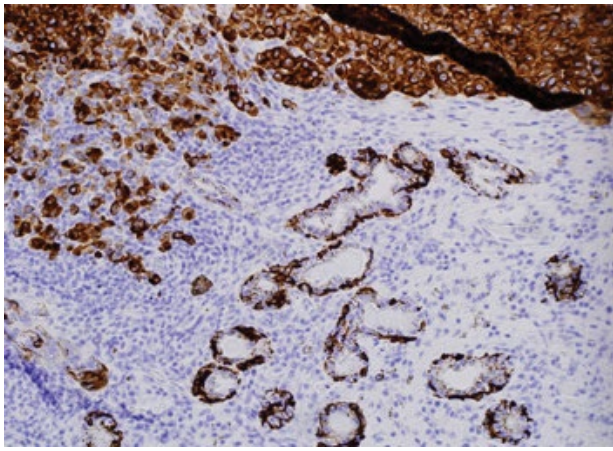

Figure 4: A) S100 stain demonstrating positivity in melanoma and adjacent glands (Immunohistochemistry, X100); B) HMB45 staining in both glandular epithelium and adjacent melanoma (Immunohistochemistry, X200); C) Reactivity for melan A in both melanoma and glands (Immunohistochemistry, X200).

reactive for these three antigens (Figure 4A, Figure 4B and Figure $4 \mathrm{C}$ ). The cuboidal cells also contained granular pigment positive by the Fontana-Masson reaction. This black pigment was most intense near the laminal surface of the cells (Figure 5). Occasional Fontana-Masson positive cells were present in the overlying mucosa near the melanoma and occasionally loose in the stroma (Figure 6).

\section{Conclusions}

Mucosal melanomas are rare and are reported to have a more aggressive behavior than their cutaneous counterparts [12-17]. Sinonasal melanomas appear to be the most common of the head and neck mucosal melanomas $[12,18]$. Sinonasal melanomas arise in a variety of sites with approximately $55 \%$ arising in the nasal cavity, $12 \%$ to $44 \%$ in the lateral wall, $8 \%$ to $20 \%$ in the nasal septum and $10 \%$ to $50 \%$ in a single sinus $[15,19,20]$. Melanomas may take a variety of cell shapes with epithelioid, plasmacytoid, undifferentiated, spindle cell, clear cell and mixed types being reported $[15,17,21]$. The mixed type is most

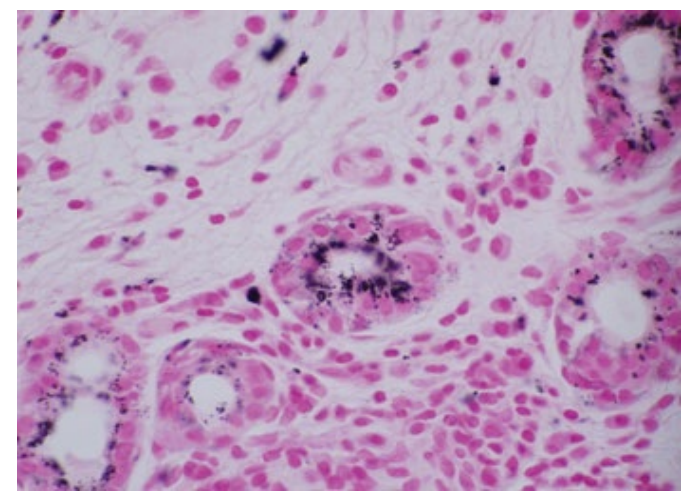

Figure 5: Fontana-Masson staining of glandular epithelium (Fontana-Masson, X600).

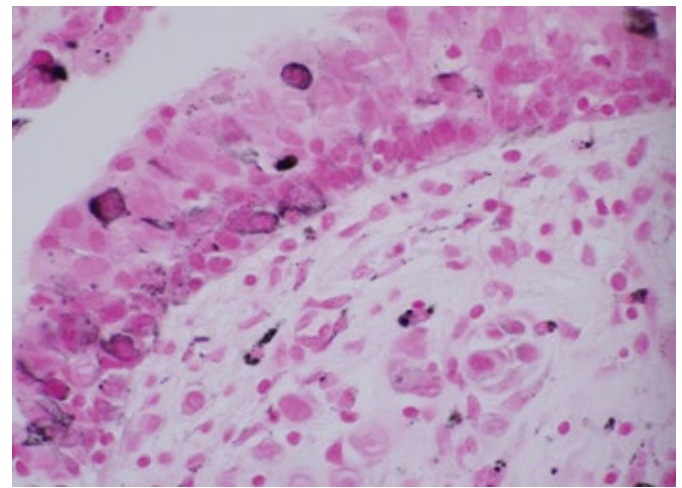

Figure 6: Focal positivity for Fontana-Masson reaction in respiratory epithelium (Fontana-Masson, X600).

common followed by the epithelial type [15]. Sinonasal melanomas frequently express S-100 protein, HMB-45 and melan $A[15,17]$. The melanoma cells in our case were predominately oval to polygonal in shape (epithelioid) and were S-100 protein, $\mathrm{HMB}-45$ and melan A reactive.

The mutational profile of mucosal melanomas including sinonasal melanomas differs from cutaneous melanomas. Unlike cutaneous melanoma, sinonasal melanomas tend to be BRAF mutation negative but show mutations in KIT $[16,17,20]$.

Sinonasal melanomas are believed to arise from neural crest cells which migrate to the nasal mucosa [17]. Zak and Lawson demonstrated melanocytes within the nasal mucosa, glands and stroma [6]. Melanocytes within mucous glands may participate in a widespread junctional change associated with melanoma [14]. The precise site of origin for sinonasal melanomas is not clear. Several reports have disclosed pigmentary abnormalities in the mucosa near sinonasal melanomas $[4,5,12,14]$. The nature of these pigment abnormalities has varied among patients with some representing intramucosal dendritic melanocytes [5], others are examples of pigment incontinence in stroma [12] and still others appear to be associated with pigment deposition in sinonasal glands [14].

The pigmented areas discrete from the malignant melanoma in our case were associated with pigment- 
ed cells in the seromucinous glands. Most prior studies have not described pigmented cells in the seromucous glands as seen in our case [15-21]. The luminal lining cells contained not only melanin pigment (positive Fontana-Masson reaction) but also were immunoreactive for HMB-45, S-100 protein and melan A suggesting the production of melanin in these cells. Earlier reports of melanotic oncocytic metaplasia demonstrated melanin pigment in the seromucinous glands but the cells were HMB-45 negative [1]. This finding suggested that the melanin pigment may only have been phagocytized by the oncocytic epithelium rather than produced in these cells. The precise site of origin for the melanoma in our patient is unclear because of the destructive nature of the neoplasm. However, the melanoma may have arisen within the mucosa or the seromucinous glands. Our case differs in several ways from prior reports of melanocytic oncocytic metaplasia. While two prior reports and our case had melan pigment within glandular epithelum, our case was positive for HMB-45 and melan A while the others were not. Additionally, the prior reports of melanotic oncocytic metaplasia occurred in Asian men [1,2] our patient was a Caucasian man. Finally, the seromucinous glands in our case did not show oncocytic change.

Our case confirms previous reports in that malignant melanoma may arise primarily within the nasal mucosa and that some of these melanomas may be associated with mucosal or sinonasal gland pigmentary disorders. Our case supports the suggestion by McClennan and Shivas that mucous or sinonasal glands can take part in a widespread activation of junctional melanocytes associated with melanoma [14]. As in prior cases, nasal melanoma may present as epistaxis or nasal obstruction. The malignancy risk for nasal pigmentation remains unknown and will require further studies to establish risk statistics.

\section{References}

1. Na JY, Kim YH, Choi YD, Lee JS (2012) Melanotic oncocytic metaplasia of the nasopharynx: a report of three cases and review of the literature. Korean J Pathol 46: 201-204.

2. Shek TW, Luk IS, Nicholls JM, Fok KO (1995) Melanotic oncocytic metaplasia of the nasopharynx. Histopathology 26: $273-275$

3. Holdcraft J, Gallagher JC (1969) Malignant melanomas of the nasal and paranasal sinus mucosa. Ann Otol Rhinol Laryngol 78: 5-20.

4. Lewis MG, Martin JA (1967) Malignant melanoma of the nasal cavity in Ugandan Africans. Relationship of ectopic pigmentation. Cancer 20: 1699-1705.

5. Cove H (1979) Melanosis, melanocytic hyperlasia, and pri- mary malignant melanoma of the nasal cavity. Cancer 44 : 1424-1433.

6. Zak FG, Lawson W (1974) The presence of melanocytes in the nasal cavity. Ann Otol Rhinol Laryngol 83: 515-519.

7. Hirakawa E, Miki H, Ohmori M, Kobayashi S, Haba R, et al. (1999) Melanin pigmented oncocytic metaplasia of the nasopharynx. Virchows Arch 434: 455-457.

8. Dong BC, Tian H, Jia XQ, Li G, Lu C, et al. (2005) Melanotic oncocytic metaplasia of the nasopharynx. Zhonghua $\mathrm{Er} \mathrm{Bi}$ Yan Hou Tou Jing Wai Ke Za Zhi 40: 549-550.

9. Uehara T, Matsubara O, Kasuga T (1987) Melanocytes in the nasal cavity and paranasal sinus. Incidence and distribution in Japan. Acta Pathol Jpn 37: 1105-1114.

10. Kondo T, Mori K, Oka S, Morinaka S (2010) Melanotic oncocytic metaplasia of the nasopharynx as a benign mimicker of malignant melanoma: a case report. Diagn Pathol 5: 5.

11. Sakaki M, Shek TW, Hirokawa M, Kashima K, Daa T, et al. (2004) Melanotic oncocytic metaplasia of the nasopharynx: a report of seven cases and review of the literature. Virchows Arch 444: 345-349.

12. Lo RH, Chang KP, Chu ST (2010) Malignant mucosal melanoma in the nasal cavity: an uncommon cause of epistaxis. J Chin Med Assoc 73: 496-498.

13. Dwivedi R, Samanta N, Agarwal S (2006) Primary malignant melanoma of the nasal cavity and paranasal sinuses: a rare cause of epistaxis in the elderly. The Internet Journal of Head and Neck Surgery 2: 1-5.

14. AA Shivas, WD Maclennan (1963) "Melanogenic metaplasia" of mucous glands. Br J Cancer 17: 411-414.

15. Zhu W, Zou B, Wang S (2016) Clinicopathological features and prognosis of sinonasal mucosal malignant melanoma: A retrospective study of 83 cases in a chinese population. ORL J Otorhinolaryngol Relat Spec 78: 94-104.

16. Lazarev S, Gupta V, Hu K, Harrison LB, Bakst R (2014) Mucosal melanoma of the head and neck: a systematic review of the literature. Int J Radiat Oncol Biol Phys 90: 1108-1118.

17. Mikkelsen LH, Larsen AC, von Buchwald C, Drzewiecki KT, Prause JU, et al. (2016) Mucosal malignant melanoma - a clinical, oncological, pathological and genetic survey. APMIS 124: 475-486.

18. Prasad ML, Busam KJ, Patel SG, Hoshaw-Woodard S, Shah JP, et al. (2003) Clinicopathologic differences in malignant melanoma arising in oral squamous and sinonasal respiratory mucosa of the upper aerodigestive tract. Arch Pathol Lab Med 127: 997-1002.

19. Vandenhende C, Leroy X, Chevalier D, Mortuaire G (2012) Sinonasal mucosal melanoma: retrospective survival study of 25 patients. J Laryngol Otol 126: 147-151.

20. Clifton N, Harrison L, Bradley PJ, Jones NS (2011) Malignant melanoma of nasal cavity and paranasal sinuses: report of 24 patients and literature review. J Laryngol Otol 125: 479-485.

21. Thompson LD, Wieneke JA, Miettinen M (2003) Sinonasal tract and nasopharyngeal melanomas: a clinicopathologic study of 115 cases with a proposed staging system. Am J Surg Pathol 27: 594-611. 